\title{
Relationship of the diaphragm to the inferior vena cava in human embryos and fetuses ${ }^{1}$
}

\author{
ANTHONYA. PEARSON, RONALD W. SAUTER, \\ and RALPH C. OLER \\ Department of Anatomy, University of Oregon Medical School, Portland, Oregon
}

\begin{abstract}
The attachment of the inferior vena cava to the diaphragm is compared with that of the oesophagus and the aorta. The development of this attachment is described in human embryos and fetuses. In older fetuses the attached border of the diaphragm splits into several layers at its junction with the inferior vena cava. The insertion of the right crus of the diaphragm into the posterior wall of the inferior vena cava is thought to have physiological significance. The left phrenic nerve supplies the left half of the diaphragm and the left component of the right crus. The right phrenic nerve supplies the right half of the diaphragm including the right component of the right crus.
\end{abstract}

This study is based on human embryos and fetuses which were sectioned serially and stained by neurological methods. These included the protargol method of Bodian (1936), the silver gelatin method of Pearson and Whitlock (1949), and other methods developed in this laboratory by the authors. The material in this study includes human specimens whose crown-rump lengths range from 10 to $111 \mathrm{~mm}$.

The general structure and development of the inferior vena cava and the diaphragm have been described in current textbooks of anatomy and embryology. However, there are certain aspects of their development, structure, and function which are not well understood. This paper is mainly concerned with the development of the attached borders of the diaphragm to the inferior vena cava and their functional significance.

\section{OBSERVATIONS}

In young human embryos of about 15 to $17 \mathrm{~mm}$ crown-rump length the inferior vena cava is seen as a venous channel passing through the liver and the primitive diaphragm to reach the right atrium. The central margin of the diaphragm is fused with the right and left lobes of the liver (Fig. 1). The walls of the inferior vena cava are not distinct from the adjacent structures of the liver and the diaphragm.

1Supported by grants from the Oregon Heart Association and USPHS 1 R01 NS08904-02
The right crus of the diaphragm, as observed sagittally in the $15-\mathrm{mm}$ embryo, is seen as a condensation of mesenchymal tissue. This mass,

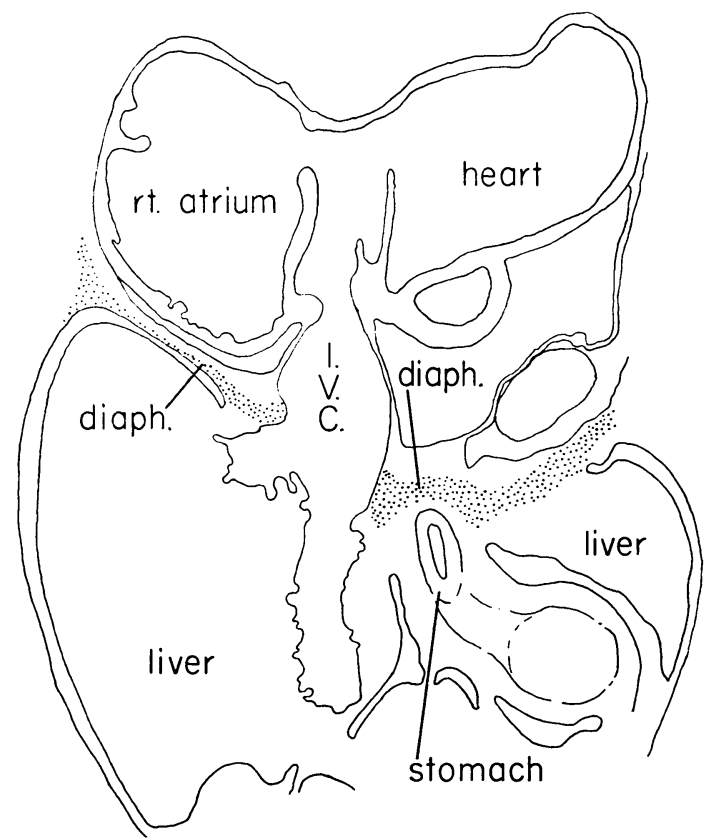

FIG. 1. A drawing from frontal sections of a 17- mm human embryo showing the path of the inferior vena cava (I.V.C) through the liver and diaphragm (diaph.) and into the heart. Note the venous valves at the superior end of the vena cava. Bodian method, $\times 28$. 
which is located to the right of the aorta, extends from the posterior body wall just superior to the posterior border of the liver and suprarenal gland and inferior to the developing pleural cavity. The developing cells line up and run cephalically and anteriorly from the posterior body wall to approach the posterior wall of the inferior vena cava. The right phrenic nerve can be seen descending on the right lateral aspect of the heart and entering the condensation of cells which it will innervate. The nerve probably acts as an inductive mechanism for the differentiation of the muscle cells.

The portion of the diaphragm anterior to the inferior vena cava shows little differentiation at this stage of development. It appears to be closely adherent to the liver and its capsule and merges with the outer layer of the anterior wall of the inferior vena cava.

In slightly older embryos of about 20 to $25 \mathrm{~mm}$ crown-rump length, the diaphragm is better developed (Fig. 2). The developing striated muscle fibres have begun to differentiate and the fibres of the phrenic nerve are prominently distributed among the muscle fibres. This is particularly well shown among the muscle fibres of the right crus.
In sagittal sections (Fig. 3) the right crus is shown extending from the vertebral column to the posterior wall of the inferior vena cava. The walls of the inferior vena cava, the ductus venosus, and the umbilical vein have begun to differentiate from the structures of the liver and the diaphragm. The attachment of the diaphragm to the lateral walls of the inferior vena cava are well shown in the transverse sections of a 24-mm human embryo (Fig. 4).

By the time the embryo has reached the $24 \mathrm{~mm}$ stage, the cells of the right crus may be identified as true myoblasts. They have not changed position but extend cephalically to the posterior wall of the inferior vena cava and appear to be inserted into the wall of this vessel. The anterior portion of the diaphragm remains unchanged.

In human fetuses of $34 \mathrm{~mm}$ crown-rump length and older, the right and left crura of the diaphragm are well developed. As the right crus is followed superiorly toward its insertion, it will be observed that it divides into right and left components which are separated by the oesophagus (Fig. 5). The majority of the muscle fibres of the right component of the right crus insert into the posterior wall of the inferior vena cava. The

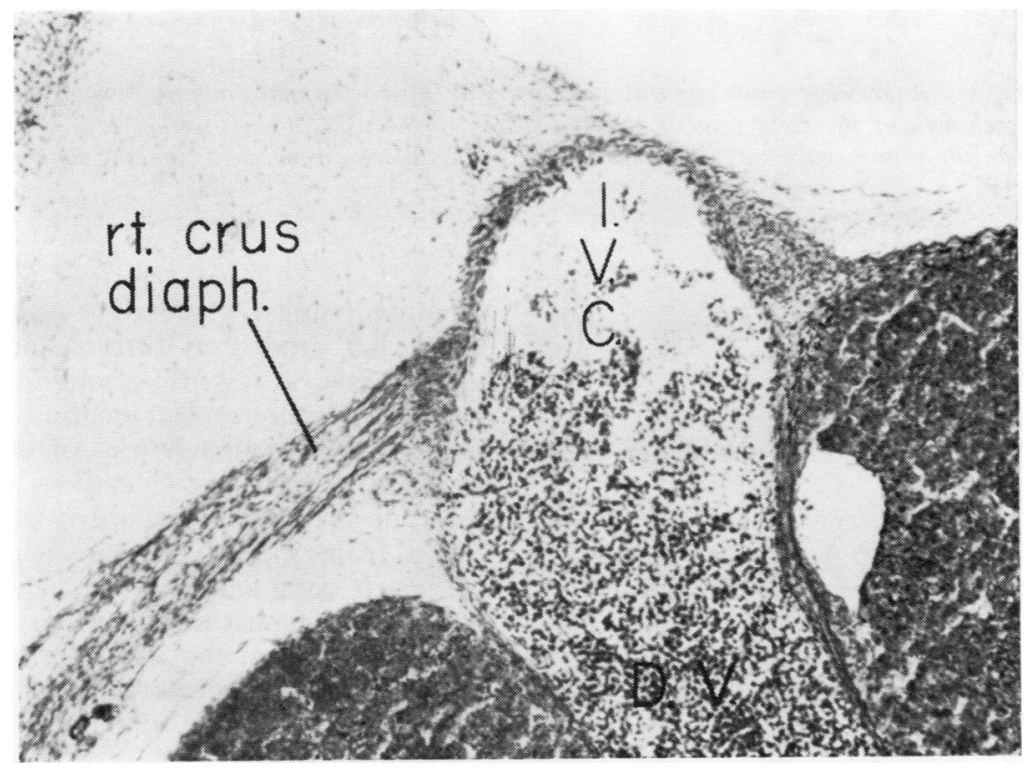

FIG. 2. A photomicrograph of a section through the liver of a 20-mm human embryo showing the junction of the ductus venosus (D.V.) with the inferior vena cava (I.V.C.). Note the attachment of the right crus of the diaphragm to the wall of the inferior vena cava. Bodian method, $\times 35$. 


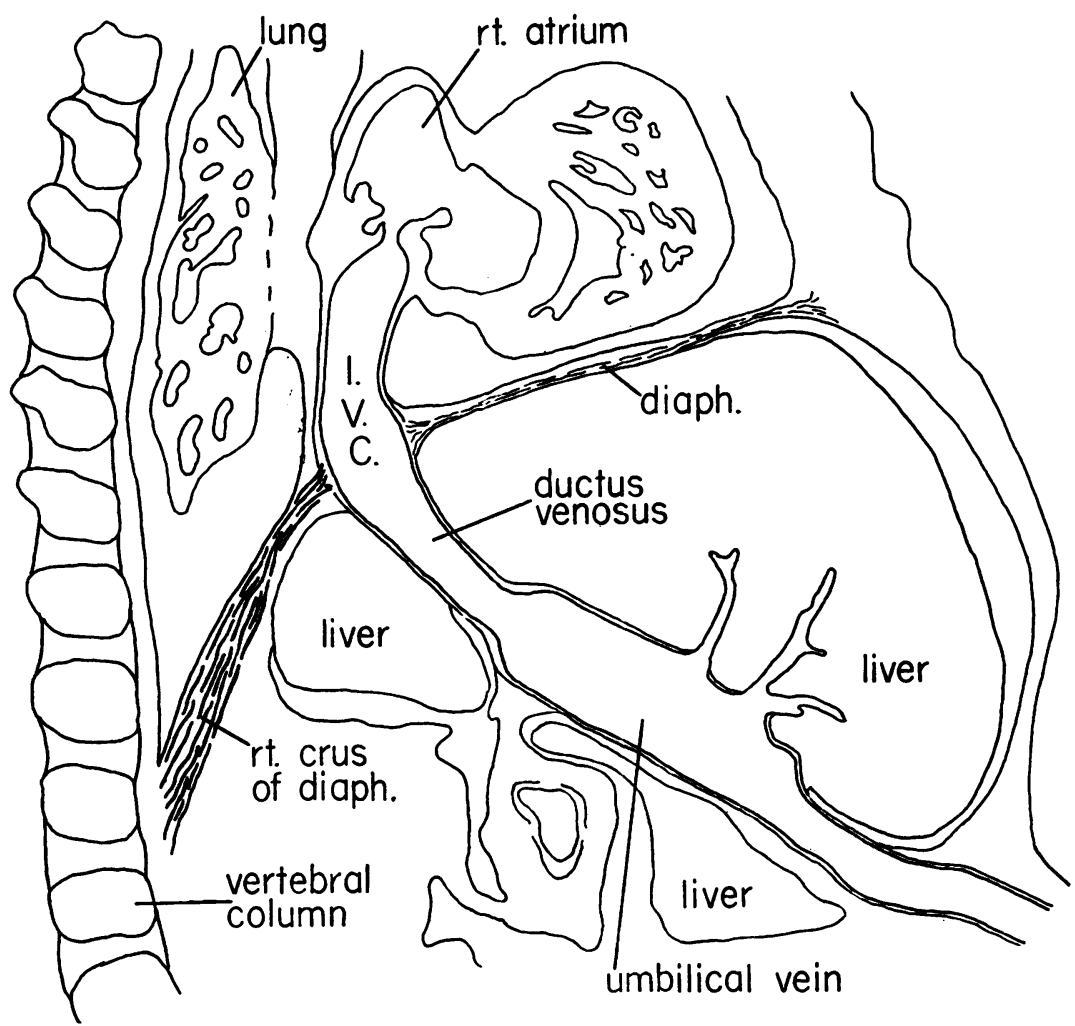

FIG. 3. A drawing from sagittal sections of a 20-mm human embryo showing the attachment of the right crus of the diaphragm to the wall of the inferior vena cava. Note the venous valves at the superior end of the inferior vena cava. Bodian method, $\times 19$.

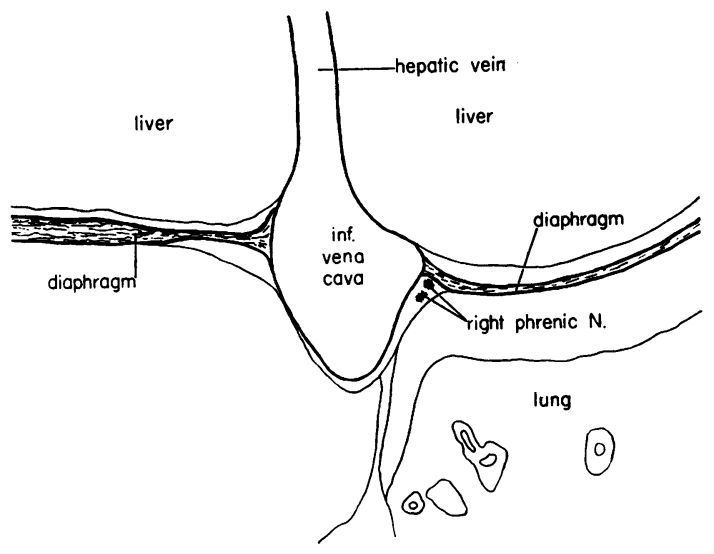

FIG. 4. A drawing of a transverse section of a 24- $\mathrm{mm}$ human embryo showing the attachments of the diaphragm to the inferior vena cava. Bodian method, $\times 25$. remaining fibres of the right crus join the fibres of the left crus, thus forming an arch over the aorta. These fibres insert into the posterior portion of the future central tendon of the diaphragm. In sections of young fetuses of this age, fibres of the right phrenic nerve can be seen among the fibres of the right component of the right crus. Fibres from the left phrenic nerve can be seen in the left component of the right crus.

During the succeeding stages, the diaphragm begins to take on the adult form by the appearance of the central tendon of the diaphragm. Instead of observing actual muscle fibres which insert into the vena cava, the muscle fibres appear to recede and a connecting link of fibrous tissue joins the right crus with the inferior vena cava. The apparent recession of the right crus is probably the result of the further development of the central tendon of the diaphragm. This sheet of 


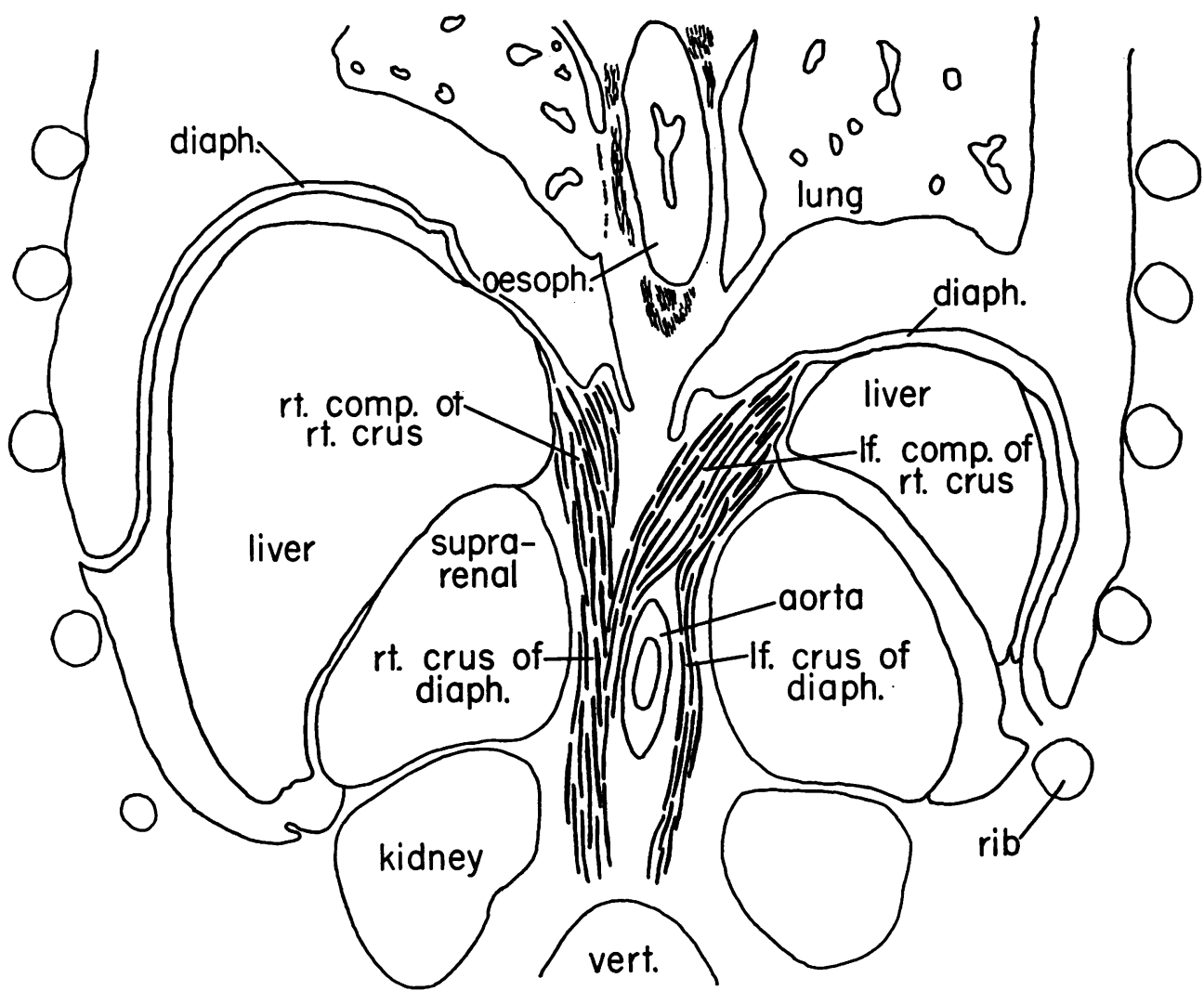

FIG. 5. A drawing from frontal sections of a 34-mm human fetus showing the right and left components of the right crus of the diaphragm. Bodian method, $\times 9.5$.

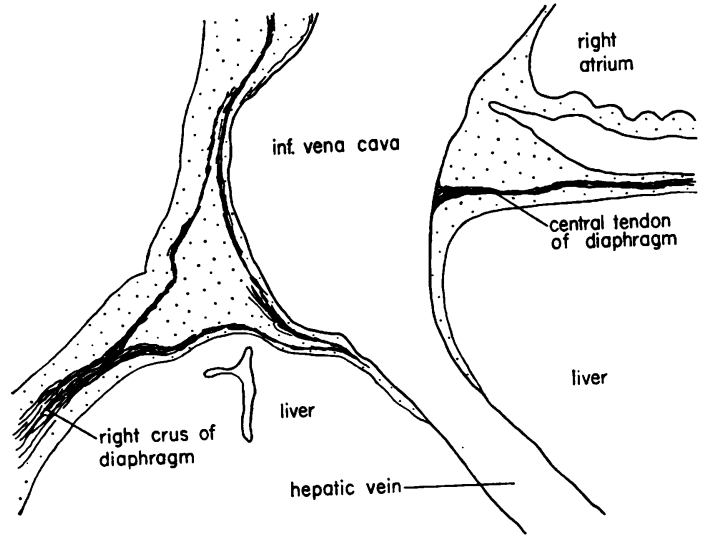

FIG. 16. A drawing from sagittal sections of a 111-mm human fetus showing the attachments of the diaphragm to the anterior and posterior walls of the inferior vena cava. Bodian method, $\times 26$. fibrous tissue extends from the muscular right crus of the diaphragm and continues superiorly and anteriorly until it reaches the inferior vena cava. Those fibres which abut against the posterior wall of this vessel turn cephalically and continue into the wall of the inferior vena cava for a short distance and make a firm attachment. Those fibres which pass wide to the inferior vena cava on either side continue ventrally and become continuous with the fibres of the anterior part of the diaphragm.

In human fetuses of more than $100 \mathrm{~mm}$ crownrump length, the central part of the diaphragm has differentiated into fibrous connective tissue which becomes the central tendon. The inferior vena cava pierces this central tendon and its walls are securely attached to the margin of the central tendon surrounding it. The attachment of the posterior and anterior walls is well shown in Figure 6. The transition of the muscular fibres to 
a fibrous tendon is shown separating into several layers. The posterior attachment illustrates the attachment of the right crus. The superficial layers of this attachment are continuous with the superficial layer of the inferior vena cava above and with the capsule of the liver below. The deeper layer is more fibrous and it is shown separating into two layers. The superior layer is shown fusing with the deeper fibrous layer of the inferior vena cava. The inferior layer fuses with or is continuous with the connective tissue in the walls of the hepatic veins, the ductus venosus, and the inferior vena cava.

Three layers can be recognized in the attached margin of the diaphragm to the anterior wall of the inferior vena cava (Fig. 6). The superior and inferior layers are composed of loose connective tissue. The superior layer is continuous with the superficial layer of the inferior vena cava and the heart. The inferior layer is continuous with the capsule of the liver. The middle layer, which is composed of denser fibrous connective tissue, fuses with the deeper denser fibrous tissue layer of the inferior vena cava.

In embryos and fetuses (Figs. 2, 4, and 6) there exists a slight enlargement of the inferior vena cava at the confluence of the ductus venosus and large hepatic veins with the inferior vena cava.

\section{DISCUSSION}

The inferior vena cava is said to be a composite structure which replaces the cardinal veins of young embryos. It is formed by the enlargement and straightening of local venous channels (Patten, 1946). This blood stream follows a venous channel through the liver and the diaphragm to reach the heart (Fig. 1). With further growth this vessel becomes better organized and it expands toward the dorsal surface of the liver. The portions of this vessel passing through the liver and the diaphragm should be referred to as the hepatic and the diaphragmatic parts of the inferior vena cava respectively. While the diaphragmatic and hepatic portions are only small parts of this vessel their significance is often overlooked. However, they are important from a functional point of view.

Early in development, the opening of the sinus venosus into the atrium is guarded on each side by a valvular fold, the right and left venous valves. The left venous valve undergoes regression and fuses with the atrial septum. A portion of the right venous valve becomes the valve of the inferior vena cava (Langman, 1963).
The valvular folds at the superior end of the inferior vena cava are relatively larger in embryos and young fetuses (Figs. 1 and 3) than they are in the new-born baby and the adult. It is quite possible that the valvular mechanism at the opening of the inferior vena cava into the right side of the heart aids in the retention of blood received from the inferior vena cava and that this function is important in the circulatory system in the embryo and the fetus.

Each phrenic nerve supplies motor fibres to its half of the diaphragm. The left half of the right crus lies to the left of the median plane and receives its nerve supply from the left phrenic nerve (Gardner, Gray, and O'Rahilly, 1969).

The anatomy of the three main orifices of the diaphragm differs structurally and functionally. The muscle fibres which surround the oesophageal opening are in the form of a sling-like loop and are derived from the right and left crura. They are thought to act as an extrinsic sphincter mechanism which aids in the control of the direction of flow in the oesophagus.

The aortic opening in the diaphragm is bounded on either side by the right and left crura and in front by the median arcuate ligament. The aortic opening is different from the caval and oesophageal openings in the diaphragm in that its posterior border is bounded dorsally by the vertebral column. The aortic hiatus is so formed that it prevents the herniation of abdominal viscera into the thorax without interfering with the flow of blood in the aorta. The caval hiatus differs from the other two openings in that it is more firmly attached to the diaphragm.

There has been wide discussion about whether the caval orifice is constricted or pulled widely open by the muscles of the diaphragm during inspiration. Franklin (1937) presents a thorough review of the early work on this problem. The structural arrangement of the right crus as it inserts into the posterior caval wall has been a point of much discussion. Keith (1902) attributed a pumping action in the inferior vena cava to contraction of the right crus during inspiration and proposed that it is an important mechanism for return of blood to the right heart. Franklin (1934), Franklin and Janker (1934, 1936), and Norhagen (1963) observed the caval region of the diaphragm in animals radiographically and described a constriction of the inferior vena cava during deep inspiration. Surgical section of the right phrenic nerve resulted in the absence of caval constriction during inspiration (Norhagen, 1963). Doppman et al. (1966) have reported that the 
constriction of the inferior vena cava occurs subdiaphragmatically in the presence of an elevated intra-abdominal pressure. However, in spite of these observations, the changes in the configuration of the caval orifice during inspiration still remain obscure.

The correlation of structure with function of the aortic and oesophageal hiatuses would lead one to search for a purpose for the uniqueness of the caval hiatus as well. It has been suggested that by virtue of the anatomical relationship of the right crus and attachments of the diaphragm into the inferior vena cava, contraction of the muscles of the diaphragm would tend to stretch the caval orifice wide and thus maintain the lumen of the inferior vena cava patent during inspiration (Last, 1954 ; Morris, 1966 ; Grant and Basmajian, 1965).

It may be concluded that, due to the attachments of the diaphragm to the wall of the inferior vena cava as described in this study, the shape or size of the lumen of the inferior vena cava can be altered by the contraction of the muscles of the diaphragm.

\section{REFERENCES}

Bodian, D. (1936). A new method for staining nerve fibers and nerve endings in mounted paraffin sections. Anat. Rec., 65, 89.
Doppman, J., Rubinson, R. M., Rockoff, S. D., Vasko, J. S., Shapiro, R., and Morrow, A. G. (1966). Mechanism of obstruction of the infradiaphragmatic portion of the inferior vena cava in the presence of increased intraabdominal pressure. Invest. Radiol., 1, 37.

Franklin, K. J. (1934). Further observations on the venae cavae of certain mammals. J. Anat. (Lond.), 68, 338.

- (1937). A Monograph on Veins. Thomas, Springfield, Illinois.

- and Janker, R. (1934). Effects of respiration upon the venae cavae of certain mammals, as studied by means of X-ray cinematography. J. Physiol. (Lond.), 81, 434.

- (1936). Respiration and the venae cavae. Further $\mathrm{X}$-ray cinematographic studies. J. Physiol. (Lond.), 86, 264.

Gardner, E., Gray, D. J., and O'Rahilly, R. (1969). Anatomy, 3rd ed. W. B. Saunders, Philadelphia.

Grant, J. C. B., and Basmajian, J. V. (1965). Grant's Method of Anatomy, 7th ed. Williams \& Wilkins, Baltimore.

Keith, A. (1902). The anatomy of the valvular mechanisms round the venous orifices of the right and left auricles, with some observations on the morphology of the heart. In Proceedings of the Anatomical Society of Great Britain and Ireland, pages ii-xxxvi. J. Anat. Physiol. (Lond.), 37 (New Series 17), ii.

Langman, J. (1963). Medical Embryology. Williams and Wilkins, Baltimore.

Last, R. J. (1954). Anatomy. Churchill, London.

Morris, H. (1966). Morris' Human Anatomy, 12th ed., edited by B. J. Anson. McGraw-Hill, New York.

Norhagen, $\AA$. (1963). Selective angiography of the hepatic veins. Acta radiol. (Stockh.), Suppl. 221.

Patten, B. M. (1946). Human Embryology. Blakiston, Philadelphia.

Pearson, A. A., and Whitlock, M. M. (1949). The use of a buffer in the silver gelatin stain for nerve fibers. Anat. Rec., 103, 581. 